\title{
Whole-genome sequencing identifies nosocomial transmission of extra-pulmonary Mycobacterium tuberculosis
}

\author{
David A. Barr ${ }^{1,2}$ and Tom A. Yates ${ }^{3}$ \\ From the ${ }^{1}$ Wellcome Trust Liverpool Glasgow Centre for Global Health Research, University of Liverpool, 70 \\ Pembroke Place, Liverpool L69 3GF, UK, ${ }^{2}$ Brownlee Centre for Infectious Disease, Gartnavel General Hospital, \\ 1053 Great Western Road, Glasgow G120YN, UK and ${ }^{3}$ Institute for Global Health, University College London, \\ 3rd floor, Institute of Child Health, 30 Guilford Street, London WC1N 1EH, UK
}

Address correspondence to Dr. D. Barr, Wellcome Trust Liverpool Glasgow Centre for Global Health Research, University of Liverpool, 70 Pembroke Place, Liverpool, L69 3GF, UK. email: david.barr@liverpool.ac.uk

We read this report with interest, ${ }^{1}$ but believe more clinical information is needed to confidently exclude airborne transmission of Mycobacterium tuberculosis.

The pulmonary nodules identified by CT chest in the index patient (case 1) may have cavitated as part of an immune reconstitution syndrome. It would be useful to know if any respiratory symptoms developed during the admission or subsequently; the timing of the original CT in relation to the immune reconstitution syndrome; if any additional chest imaging occurred; and if respiratory specimens were obtained for mycobacterial culture at any stage.

Patients with HIV infection may be sputum culture positive despite a normal chest x-ray. ${ }^{2}$ Positron Emission Tomography Computed Tomography (PET-CT) scanning of asymptomatic individuals with 'latent TB' can reveal appearances similar to those seen in active disease. ${ }^{3,4}$ Serial imaging of pulmonary TB shows the disease to be highly dynamic. ${ }^{5}$ A significant proportion of 'extra-pulmonary' TB cases have concomitant pulmonary disease. ${ }^{6}$

There have been previous reports of $M$. tuberculosis transmission during aerosol generating procedures, including the irrigation of abscesses. ${ }^{7}$ Transmission via direct inoculation ${ }^{8}$ and ingestion are also described-here the latter is plausible, given the site of disease in case 2 .

A better mechanistic understanding of which procedures are potentially aerosol generating might help guide practice.
Ruling-out pulmonary disease is non-trivial, particularly in the context of HIV co-infection. Most transmission of M. tuberculosis is probably from individuals with pulmonary disease not yet on effective treatment (largely undiagnosed). ${ }^{9}$ This should be the focus of global efforts to prevent nosocomial transmission.

\section{Funding}

DAB is funded through a Wellcome Trust PHD studentship grant ref 105165/Z/14/A.

Conflict of interest: None declared.

\section{References}

1. Walker TM, Crook DW, Peto TEA, Conlon CP. Whole-genome sequencing identifies nosocomial transmission of extrapulmonary M. tuberculosis. QJM 2016; 109:819-20.

2. Pepper T, Joseph P, Mwenya C, McKee GS, Haushalter A, Carter $A$, et al. Normal chest radiography in pulmonary tuberculosis: implications for obtaining respiratory specimen cultures SUMMARY. Int J Tuberc Lung Dis 2008; 12:397-403.

3. Barry CE, Boshoff HI, Dartois V, Dick T, Ehrt S, Flynn J, et al. The spectrum of latent tuberculosis: rethinking the biology and intervention strategies. Nat. Rev. Microbiol 2009; 7:845-55. 
4. Esmail H, Lai R, Lesosky M, Wilkinson K, Graham C, Coussens $A$, et al. Characterization of progressive HIV-associated tuberculosis using 2-deoxy-2-[18F]fluoro-D-glucose positron emission and computed tomography. Nat Med 2016; 22:1090-5.

5. Malherbe ST, Shenai S, Ronacher K, Loxton AG, Dolganov G, Kriel M, et al. Persisting positron emission tomography lesion activity and Mycobacterium tuberculosis mRNA after tuberculosis cure. Nat Med 2016; 22:1094-100.

6. Sterling TR, Pham PA, Chaisson RE. HIV infection - related tuberculosis: clinical manifestations and treatment. 2010; 50:223-30.
7. Beggs CB, Noakes CJ, Sleigh PA, Fletcher LA, Siddiqi K. The transmission of tuberculosis in confined spaces: An analytical review of alternative epidemiological models. Int J Tuberc Lung Dis 2003; 7:1015-26.

8. Fennelly KP, Jones-López EC. Quantity and quality of inhaled dose predicts immunopathology in tuberculosis. Front Immunol 2015; 6:1-13.

9. Escombe AR, Huaroto L, Ticona E, Burgos M, Sanchez I, Carrasco L, et al. Tuberculosis transmission risk and infection control in a hospital emergency department in Lima, Peru. Int $J$ Tuberc Lung Dis 2010; 14:1120-6. 